\title{
Channel Estimation using Kalman Filter for MIMO-OFDM Communication System
}

\author{
G.Rajender, Tipparti.Anilkumar
}

\begin{abstract}
With the advancement of remote correspondence, the confinement of sign estimation under channel variation condition and their belongings were expanding. Different systems were proposed in past for the improvement of sign estimation effectiveness dependent on reference data utilizing versatile, visually impaired or semi visually impaired methodologies. Where visually impaired and semi visually impaired are seen to beat the versatile based methodologies, further upgrades are still on research to improve the productivity with least time union. To accomplish this goal, estimation calculations in time, recurrence and time-recurrence area were created. These methodologies attempt to accomplish the productivity targets by either expanding the estimation recursion or restricting the mistake likelihood. This paper exhibits a methodology for accomplishing improved estimation proficiency with least time assembly and lesser mistake likelihood, in MIMO correspondence framework utilizing the kalman filtration approach. A ghastly estimation rationale dependent on vitality of the sign range is made.
\end{abstract}

Keyword: Signal Estimation, time variant channel condition, MIMO communication, spectral coding.

\section{INTRODUCTION}

Remote direct are getting different in nature. Direct deviation in correspondence procedure has been successful in channel estimation, which is reliant on the channel vulnerability. The recovery of sign under channel flawed conditions. In various methodology of estimation, input estimators are utilized as an ideal estimation rationale, where a criticism mistake is spread to get the estimation, The estimation rationale work utilizing a Where the channel estimation is being completed and refreshed to acquire a gauge. In the MIMO-OFDM framework, the estimation utilizing notwithstanding, with the methodology of expanding correspondence and administrations, we need the customary models of the correspondence of the information trade to improve. To accomplish the best execution of this framework, various strategies were created in later past. Under the direct defect condition in [1] a recurrence specific rationale is created, where a pilot base estimation approach is created to appraise the sign under multipath condition. Channel works for direct following in the correspondence framework and encoding showing gives an adaptable methodology. The investigation is a total assessment of the divert increase and stage in the correspondence framework. It has been put early that input estimators pursue the all-encompassing stage clamor. It is seen that the all-inclusive separating methodologies are

Revised Version Manuscript Received on 10, September 2019.

G.Rajender, Associate Professor, ECE Department, CMRIT Hyderabad, Telangana, India.(Email: rajenderg2012@gmail.com)

Dr.Tipparti.AnilKumar, Professor, ECE Department, CMRIT Hyderabad, Telangana, India.(Email: tvakumar2000@yahoo.co.in) increasingly steady in the estimation of stage following clamor. A comparative methodology utilizing the semi-dazzle way to deal with gauge the direct is also sketched out. 'It is utilized for continuous estimation in sensible estimation dependent on input utilizing squares of information bits. Legitimate gauge was created dependent on a unique square, considering the instance of including the channel rapidly and the condition of divert decent variety in the framework is sketched out in [2].

To accomplish the estimation at quicker rate an answer for the channel estimation approach dependent on the criticism channel is illustrated. The state determines move coefficient dependingon whether the rationale of the right worth is constrained to a period changing condition. So as to accomplish the goal of the client's development, another worry moving based estimation for the MIMO-OFDM framework is proposed in [3]. A guess technique to appraise the channel at various speed of development in the moderate, quick and medium arrangement is watched. It is intended to channel the exhibition of the criticism channel uncovered that the sign contained in the development case is more meddled contrasted with static model. The estimation execution depends on the divert impact saw in the correspondence procedure. The effectiveness of coding of a channel estimator is worried about the channel thought. For the estimation of sign, in [4] a motivation reaction of channel impact is utilized, where connection rationale is created for a correlative methodology for transmit and get receiving wire. A methodology of coordinated wiener channel is utilized for the enhancement of direct estimation in a period recurrence space. The estimation execution under exceptionally changing versatile units for Time ward channel impact. For the improvement of estimation execution in MIMO-OFDM framework, a symmetric methodology was delineated in [5].

The estimation approach is characterized to limit the MSE, power and obstruction dependent on DFT approach. The estimation of channel impact utilizing even estimation model was utilized for the MSE and power misfortune minimization. In [6] to evaluate the SCM channel for MIMO-OFDM frameworks with zero-fill is characterized with an unmistakable properties. State check is basic and the loosest of the segment is distributive mode. it tends to be applied to the instance of transmission reception apparatuses specifically cases. Through the assessment of recreation, the mistake rate execution is seen in the recovered bits are low to medium in estimation zone. To decide way nearer to the best estimation per second round of the $\mathrm{CFO}$ and advances in 
putting OFDM framework is proposed. Adjusting gives top of the line replication calculation to fit to evaluate the great track that the conventional technique for the examination scales. It works in the second round adjusting route in improving the frequencies and channels to follow estimation when contrasted with the primary structure. In [7] diverting long haul estimator properties is processed through a sub area mapping calculation through space-time designs in the channel. Then again, factor blurring can be followed utilizing the methods utilized for transient blur channel rationality.

Specifically, it was picked in MIMO-OFDM dependent on a BISM condition Turbo reference model for execution assessment as far as bit-mistake rate. Plan has been put to agree with the semi-daze plan to evaluate the time channels for frameworks and modules dependent on vectors. In semi-daze coding, the activity was performed through three usable stages, (1) ascertaining the time distinction between the most extreme addition in different channel blurring, (2) minute sedimentation time calculation to discover an area in the channels, and (3) Reach of the channel reaction. In this work, another strategy is proposed to assess the exhibition of the MIMO-OFDM frameworks through discretionary and covering variable recurrence channels. Results are estimated based on likely mistake bits when the coded is traded over the channel. We centre around the type of coding framework for most channel criteria dependent on OFDM with BISM innovation. The examination dependent on the methodology of the channel decent variety is utilized and can be reached out to any of the squares size.

\section{CORRESPONDENCE FRAMEWORK MODEL \& RESULTS}

In fast portable radio correspondence frameworks, the plan of a remote channel which goes about as a medium will represent a more noteworthy test. As per figure.1, the sign will be experienced different kinds of debasements when it was transmitting through the channel. In this manner, the sign got at the beneficiary is the mix of unique sign, the sign of different clients. This consolidated likewise having a self-assertive lessening impact and defers impact. Since, there will dissipate, there is an opportunity to get numerous duplicates of the same sign at the beneficiary because of the snags. The invert connection has non concurrent nature. I.e., different sign got at beneficiary will have different time-balances concerning the reference timing discretionarily, as appeared in figure. At that point the got sign change is taken from pass band to base band, demodulation, and digitization pursued by discovery and unravelling into data bits.

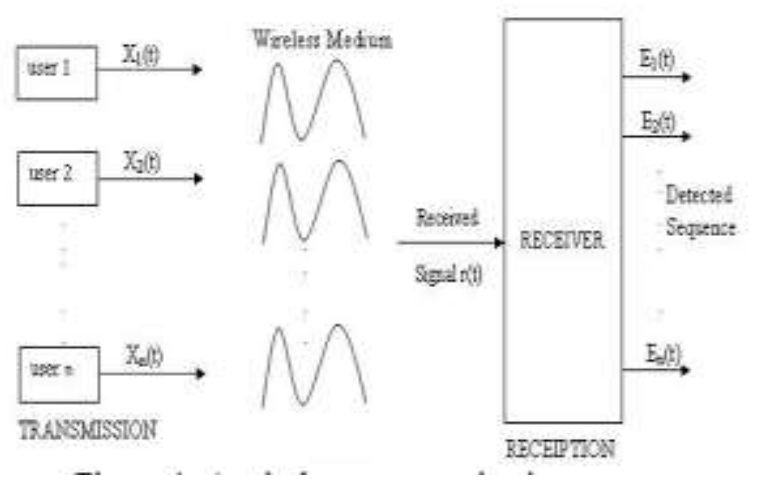

\section{Fig1 -A wireless communication system}

\section{RESULTS ANALYSIS}

Iterative Doppler helped channel estimation with PIC-DSC obstruction retraction conspire for the standardized Doppler spread of 0.025 .This is comparable to a client moving at speed of $81 \mathrm{~km} / \mathrm{h}$ with the LTE parameters. The standardized Doppler spread of 0.025 , the channel varieties are moderate, and subsequently, even a straightforward channel estimation procedure performs satisfactorily.

The reproduction results demonstrate that this methodology marginally improves the SER execution of the proposed calculation for the standardized Doppler spread of 0.025 . Be that as it may, the utilization of introduction diminishes the throughput effectiveness of the framework.

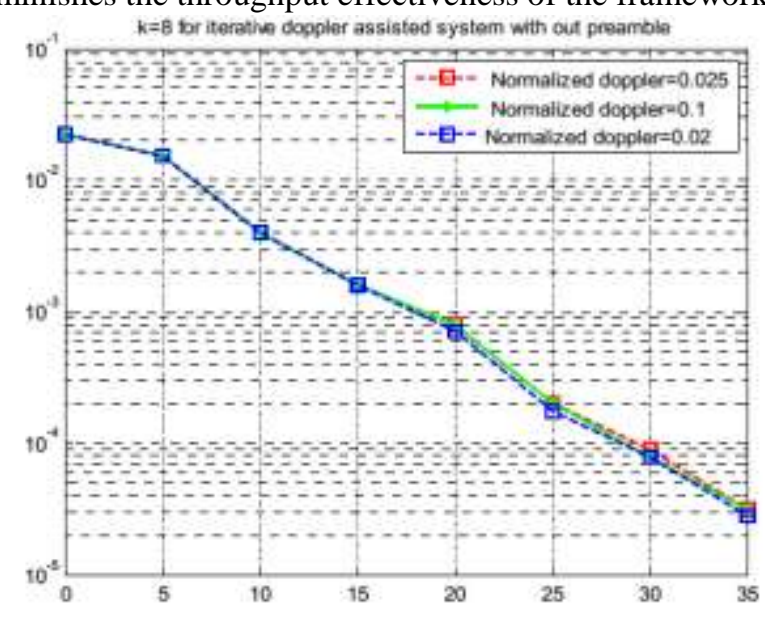

Fig 2: Comparison of the SER performance of the proposed iterative Doppler-assisted channel estimation with and without preamble.

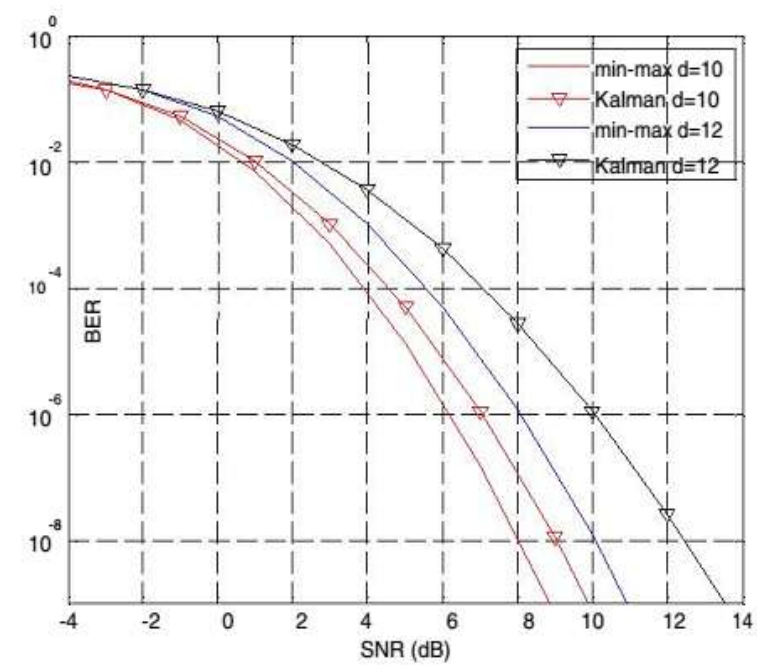

Fig 3 . BER v/s SNR Performance in randomized 2interference

Execution of a framework with QPSK balance and, Obstruction randomization with randomized channel technique and fixed postpone spread for $=1$ is exhibited in figure. The offered information rate (kbps) ' $\mathrm{d}$ ' is changed for the created methodology 


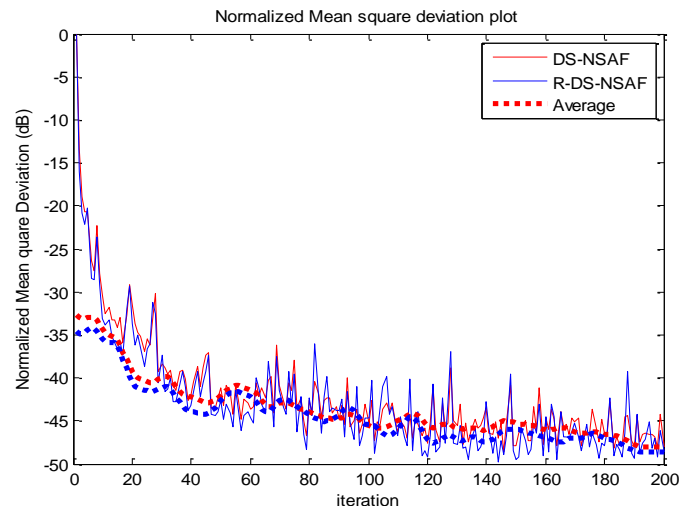

Fig 4 . Normalized MSD for the two methods at $N=8, \sigma$ $=0.8$

For the estimation of the first sign, the clamor influenced test is considered for 8 channels. The thickness of clamor influenced groups is appeared in figure 5. On account of clamor influenced signal, the deteriorated groups comprise more magnitudal mutilation, than the first example. In reference to these groups estimation is completed. The Got perception for this case is sketched out in figure. The standardized MSD for the two techniques at $\mathrm{N}=8$ is as sketched out in figure. It could be seen that, with the expansion in the estimation of ' $\mathrm{N}$ ', the MSD is decremented for the Randomized methodology. As the estimation in such case is extended all groups, the mistake likelihood will be less. Anyway on account of DS, the estimation is done more than two progressive groups as it were.

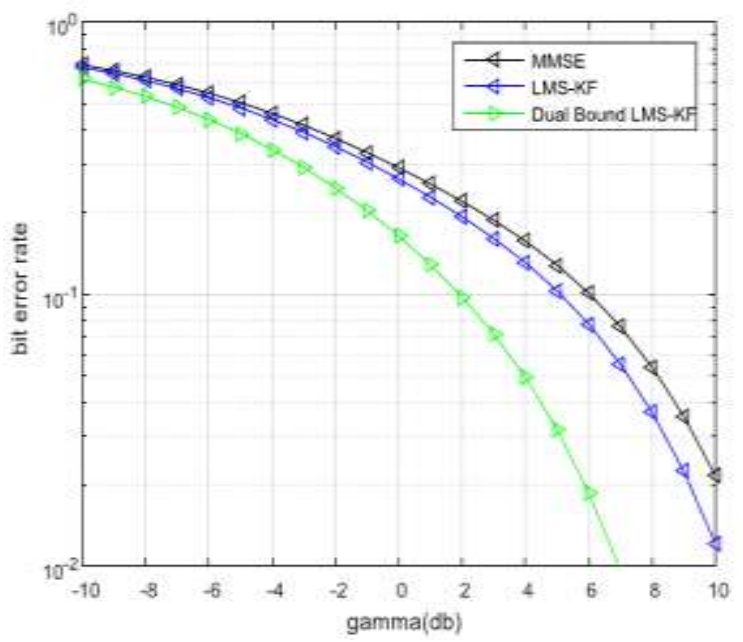

Fig 5 . BER execution of the created methodology contrasted and regular estimation draws near

Figure outlines the BER execution for various advance size qualities ideally inferred for various channel estimation to be 5 for $M=21$ coming about agreeable union. It is seen that the BER esteems are altogether improved through iterative estimation of flawlessly instated Double bound LMS-KF calculation in contrast with that of regular approaches.

\section{CONCLUSION}

The Mistake execution of the created methodology is seen to be limited under the diverse channel condition utilizing the customary kalman and min-max estimation approach. The general execution was demonstrated to be ideal under channel decent variety condition, for arbitrary just as facilitated impedance model. The mistake execution approves the use of proposed min-max approach when contrasted with ordinary kalman filtration. New iterative Doppler helped channel estimation with the PIC-DSC impedance crossing out plan for high portability MIMO-OFDM frameworks. In the proposed strategy, the remote channel has been evaluated by utilizing the Doppler spread data, pilot images, and gauges of the information images at the beneficiary. Each time-space channel coefficient is communicated as a weighted introduction between two chose time-area channel coefficients alluded to as time-area markers. These double cross space markers are chosen such that they have most extreme relationship with the individual channel coefficient. The insertion loads are planned dependent on Doppler spread data at the beneficiary.

\section{REFERENCES}

1. Hani Mehrpouyan, Ali A. Nasir, Steven D. Blostein, Thomas Eriksson, George K. Karagiannidis, and Tommy Svensson, "Joint Estimation of Channel and Oscillator Phase Noise in MIMO Systems", IEEE Transactions on Signal Processing, Vol.60,(9),pp.4790 - 4807,2014.

2. G Rajender,Dr T Anil Kumar and Dr K Srinivasa Rao"Estimation And Performance- Kalman Based Approach: A Review" Ieee International Conference On Communication And Signal Processing (Iccsp-16/IEEE) April 6-8, 2016, Pp: 1145-1151.

3. G Rajender,Dr $\mathrm{T}$ Anil Kumar and Dr K Srinivasa Rao"Recursive State Optimization Using Min-Max Approach For Channel Estimation In Mimo System" IEEE International Conference On Global Trends In Signal Processing, Information Computing And Communications (Icspicc-2016) Dec 22-24, 2016, Pp: 272-277.

4. Jinesh P. Nair and Ratnam V. Raja Kumar, "Ideal Superimposed Training Sequences for Channel Estimation in MIMO-OFDM Systems", Hindawi Publishing Corporation, EURASIP Journal on Advances in Signal Processing, pp.13, Springer, 2010.

5. Eric Pierre Simon, Laurent Ros, Hussein Hijazi, Jin Fang, Davy Paul Gaillot, Marion Berbineau, "Joint Carrier Frequency Offset and Fast Time-fluctuating Channel Estimation for MIMO-OFDM Systems", IEEE Transactions on Vehicular Technology, Institute of Electrical and Electronics Engineers, Vol., 60, pp.955-965, IEEE, 2011.

6. G Rajender,Dr T Anil Kumar and Dr K Srinivasa Rao" Kalman Stabilization Under Channel Diversity In Mimo-Ofdm System" In International Journal Theoretical And Applied Informatio Technology(Jatit)-30 ${ }^{\text {th }}$-June-2017.Vol.95.No12,Pp:26882697.

7. $\mathrm{G}$ Rajender,Dr $\mathrm{T}$ Anil Kumar and $\mathrm{Dr} \mathrm{K}$ Srinivasa Rao"Dual Bound Kalman Filter For Signal Estimation In Multipath Fading For MIMO-OFDM communication System" International Journal Of Innovative Technology And Exploring Engineering (Ijitee) Issn: 2278-3075, Volume-8 Issue-7 May, 2019, Pp-690-696 